\title{
Vitrectomy and Microperimetry of an Accidental Macular Hole Caused by Nd:YAG Laser
}

\author{
Yung-Jen Chen \\ Department of Ophthalmology, Chang Gung Memorial \\ Hospital - Kaohsiung Medical Center, Chang Gung University College \\ of Medicine, Kaohsiung, Taiwan, ROC
}

\section{Key Words}

Accidents · Lasers · Laser injury · Macular hole · Microperimetry · Vitrectomy

\begin{abstract}
A 31-year-old man sustained a 1,064-nm Q-switched Nd:YAG laser injury to his left eye. One month after the injury, the fundus and optical coherence tomography (OCT) examination demonstrated a full-thickness macular hole of approximately $820 \mu \mathrm{m}$ in diameter. After vitrectomy and internal limiting membrane peeling, OCT showed closure of the hole and a marked thinning of the foveal depression. After 12 months of follow-up, his visual acuity improved from 20/60 to 20/20. The MP1-microperimeter demonstrated increased retinal sensitivity in the area of the previous macular hole and its adjacent region and improvement of fixation from a relatively unstable status to a stable status. The macular hole remained closed 24 months postoperatively with the best corrected visual acuity $20 / 20$. Our results suggest that vitrectomy can improve the visual function when a macular hole is caused by Nd:YAG laser injury. The improvement in the visual function includes not only visual acuity but also retinal sensitivity and fixation stability that are obtained by using the MP1-microperimeter.
\end{abstract}

\section{Introduction}

The Q-switched Nd:YAG laser may cause photodisruption at the injury site, which occasionally results in a macular hole [1-7]. Thach et al. [1] described 5 eyes of 4 patients with macular holes caused by accidental Q-switched Nd:YAG laser injury. In 1 of these eyes, the macular hole resolved spontaneously. Long-term delayed surgery or observation in these cases may result in a poor or limited outcome [1,7]. However, the indications and roles of surgery have not been clearly defined. Vitrectomy with gas tamponade has been shown to improve the visual acuity in patients with Q-switched Nd:YAG laser-induced macular holes $[2,5,6]$. 
We report a case of a macular hole caused by accidental exposure to an Nd:YAG Q-switched laser. Preoperative and postoperative microperimetry measurements were used for functional analysis. Microperimetry was performed using the MP1microperimeter (Nidek Technologies, Albignasego, Italy). We used the following parameters: a fixation target consisting of a single cross, $2^{\circ}$ in diameter; a white, monochromatic background at 4 asb (apostilb), a stimulus size Goldman III, with projection time of $200 \mathrm{~ms}$; a customized radial grid of 33 stimuli covering $20^{\circ}$ (centered onto the fovea). The starting stimulus light attenuation was set at $10 \mathrm{~dB}$. A 4-2-1 double staircase strategy was used with an automatic eye tracker that compensates for eye movements.

\section{Case Report}

The institutional review board approved the retrospective chart review study. A 31-year-old electronics technician who had undergone bilateral laser-assisted in situ keratomileusis surgery 7 years before accidentally sustained a laser injury to his left eye while repairing and aligning a 1,064-nm $\mathrm{Nd}$ :YAG laser. The Q-switched laser pulse energy was approximately $10 \mathrm{~mJ}$ and the pulse duration was 5-20 ns. After a single shot of the laser, he immediately noticed a small central blind spot and floaters in his left eye. At the time of examination within $24 \mathrm{~h}$ after injury, his best corrected visual acuity (BCVA) was 20/20 in the right eye and 20/200 in the left eye. The examination of the left fundus revealed a marked white juxtafoveal burn with a small retinal hemorrhage and vitreous hemorrhage (fig. 1a). The optical coherence tomography (OCT) (Stratus OCT 3; Carl Zeiss, Dublin, Calif., USA) examination revealed increased thickness in the foveal area and acoustic shadows caused by the vitreous hemorrhage (fig. 1d). One month later, his BCVA was 20/60 in the left eye. Fundus examination revealed a macular hole with pigment clumping at its base and elevated edges (fig. 1b). In addition, the OCT demonstrated a full-thickness macular hole of approximately $820 \mu \mathrm{m}$ in diameter with cystoid changes adjacent to the hole (fig. 1e). The MP1-microperimeter demonstrated a retinal sensitivity of $0 \mathrm{~dB}$ over the macular hole and decreased retinal sensitivity in the adjacent area (fig. 2a). The fixation was relatively unstable with 51 and $97 \%$ of the preferred fixation points located within a $2^{\circ}$ - and $4^{\circ}$-circle area centered in the fovea, respectively. The fixation target of a single cross was positioned at the preferred fixation point as the foveal center because the center of the foveal avascular zone could not be determined due to the large macular hole. The central fixation location was poor and $46 \%$ of the preferred fixation points were located within the $2^{\circ}$-circle area centered in the fovea. Surgical intervention was advised because of the unlikely possibility of the spontaneous closure of such a large macular hole. The patient underwent pars plana vitrectomy with the creating of posterior vitreous detachment. We injected $0.1 \mathrm{ml}$ of indocyanine green (ICG) at a concentration of $2.5 \mathrm{mg} / \mathrm{ml}$ in the temporal macular region without direct staining of the hole. The ICG was immediately removed from the vitreous cavity with a vitreous cutter. A bent 25 -gauge needle was used to create a small temporal incision 2 disc diameters (DD) away from the fovea. The internal limiting membrane (ILM) was then peeled in the area around the macula (2 DD), and the eye was filled with a $16 \% \mathrm{C}_{3} \mathrm{~F}_{8}$ mixture. The patient was advised to maintain a face-down position for 2 weeks.

The closure of the macular hole was observed within 1 month postoperatively. Fundus examination revealed closure of the hole associated with pigment clumping

(fig. 1c). The OCT showed a marked thinning of the foveal depression and high reflectivity in the subfoveal area with an acoustic shadow (fig. 1f). His BCVA improved to 20/30 in 3 months and to 20/20 in 12 months postoperatively. The MP1-microperimetry 12 months after surgery demonstrated increased retinal sensitivity in the area of the previous macular hole and its adjacent region (fig. $2 \mathrm{~b}$ ). The fixation of the preferred fixation points located within a $2^{\circ}$ - and $4^{\circ}$-circle area centered in the fovea was stable (98 and $100 \%$, respectively). The fixation location was predominantly eccentric and $0 \%$ of the preferred fixation points were located within the $2^{\circ}$-circle area centered in the fovea. The postoperative preferred fixation position was located at the base of the previous macular hole and was different from the preoperative position (fig. 2b). The macular hole remained closed 24 months postoperatively with the BCVA 20/20. 


\section{Discussion}

Surgical intervention was advised in our case because of the unlikely possibility of spontaneous closure due to the size of the macular hole (approximately $820 \mu \mathrm{m}$ in diameter) (fig. 1b). Yet, an anatomic closure of a macular hole as big in size as noted in our case can be achieved after vitrectomy and peeling of the ILM. However, a marked thinning of the foveal depression was noted after surgery (fig. 1f). The location and severity of the damage of the central fovea tissue may limit the potential improvement in the visual outcome after surgery. In our case, the macular hole was located predominantly in the juxtafoveal area. The preoperative visual acuity was $20 / 60$, which may explain the good improvement of visual acuity of 20/20 after 24 months of postoperative follow-up.

The Amsler grid examination has been used to evaluate the extension of central scotoma and metamorphopsia caused by a laser-induced macular hole $[8,9]$. The MP1-microperimeter is a fundus perimeter, which allows for a completely automatic determination of retinal sensitivity and fixation and correlates exactly to the characteristics of the fundus. In this study, MP1-microperimeter after 12 months of postoperative follow-up showed that the retinal sensitivity was increased in the region of the previous macular hole and its adjacent area. The fixation improved from a relatively unstable status to a stable status. The fixation location changed from a poor central fixation to a predominantly eccentric fixation, which may partially be due to the poor preoperative determination of the foveal center and the fixation target positioned at the preferred fixation point as the foveal center preoperatively. However, the postoperative preferred fixation position was different from the preoperative position and was located at the base of the previous macular hole.

Our results suggest that vitrectomy can improve the visual function when a macular hole is caused by Nd:YAG laser injury. The improvement in the visual function includes not only visual acuity but also retinal sensitivity and fixation stability that are obtained by using MP1-microperimeter. 


\begin{tabular}{|c|c|c|c|}
\hline Case Reports in & $\begin{array}{l}\text { Case Rep Ophthalmol 2010;1:80-84 } \\
\text { DOI: } 10.1159 / 000321631\end{array}$ & $\begin{array}{l}\text { Published online: } \\
\text { October 16, } 2010\end{array}$ & $\begin{array}{l}\text { @ } 2010 \text { S. Karger AG, Basel } \\
\text { ISSN } 1663-2699 \\
\text { www.karger.com/cop }\end{array}$ \\
\hline
\end{tabular}
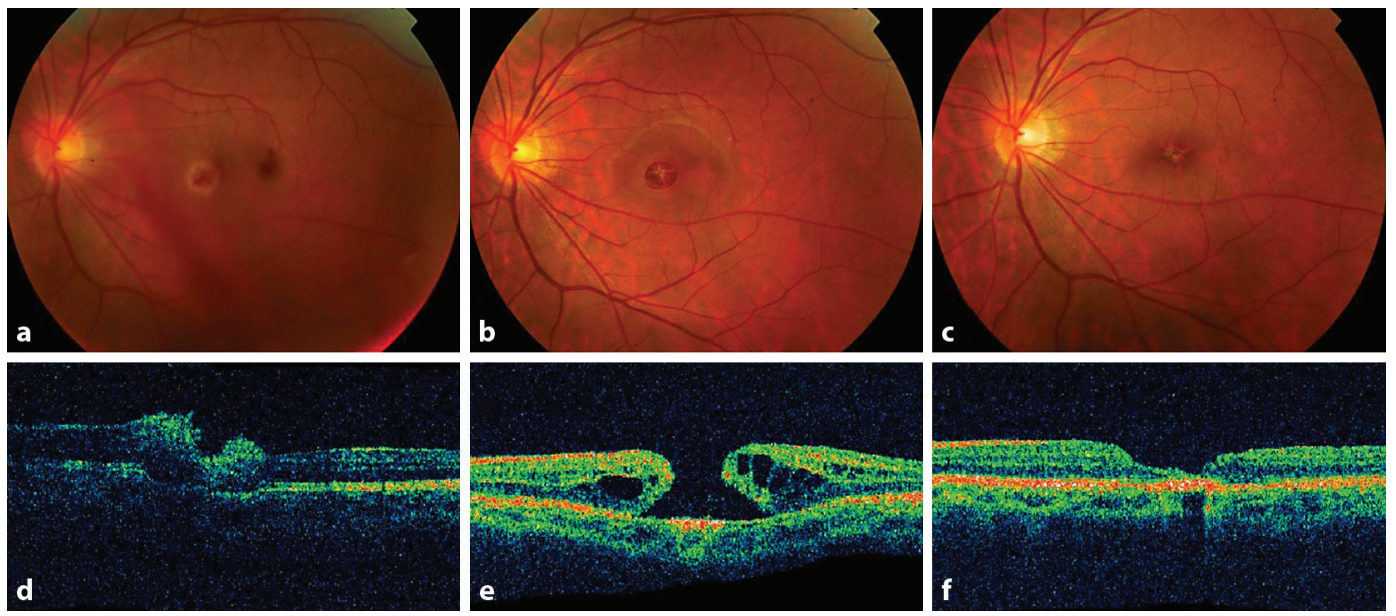

Fig. 1. Color fundus photographs (a-c) and horizontal optical coherence tomography (d-f) images from the left eye. a Within 24 hours after injury, color fundus photograph revealed a marked white juxtafoveal burn with a small retinal hemorrhage and vitreous hemorrhage. b One month after injury, color fundus photograph revealed a macular hole with pigment clumping at its base and elevated edges. c Three month after vitrectomy, color fundus photograph revealed closure of the macular hole associated with pigment clumping. d Within 24 hours after injury, optical coherence tomography showed thickness in the foveal area and acoustic shadows caused by the vitreous hemorrhage. e One month after injury, optical coherence tomography showed a full-thickness macular hole with cystoid changes adjacent to the hole. $\mathbf{f}$ Three month after vitrectomy, optical coherence tomography showed a marked thinning of the foveal depression and high reflectivity in the subfoveal area with an acoustic shadow.
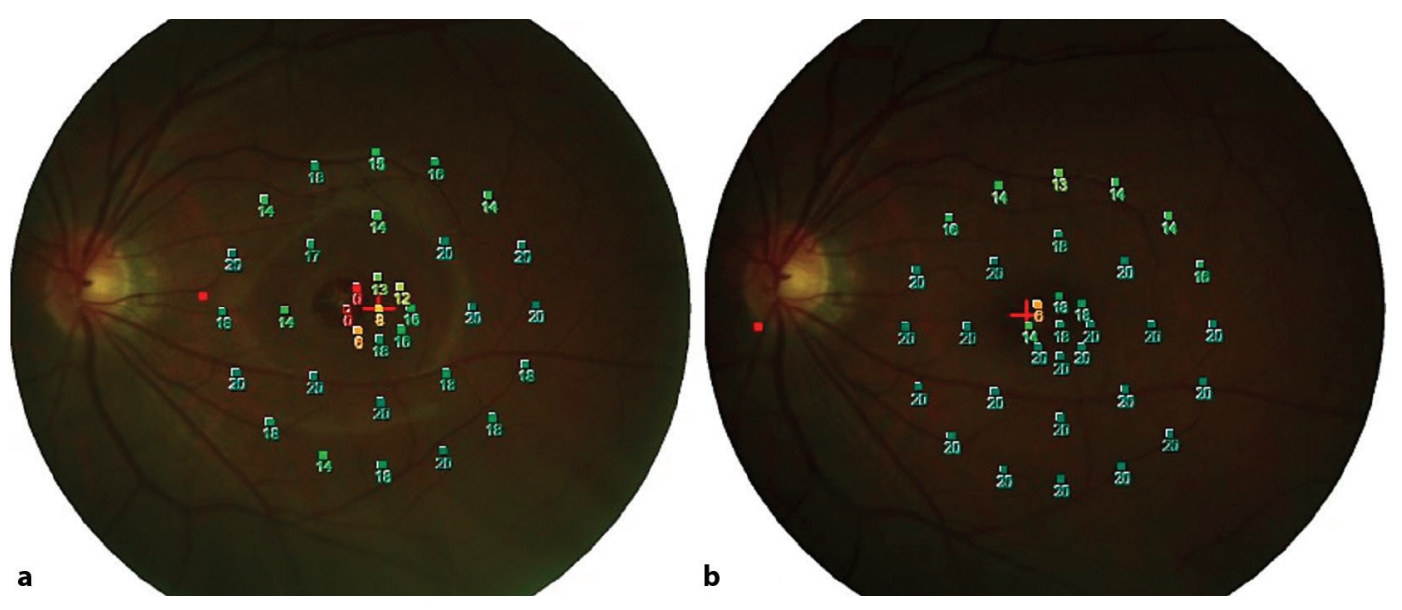

Fig. 2. MP1-microperimeter examination of the left eye. a One month after injury, examination demonstrated a retinal sensitivity of $0 \mathrm{~dB}$ over the macular hole and decreased retinal sensitivity in the adjacent area. The fixation target of a single cross was positioned at the preferred fixation point as the foveal center because the center of the foveal avascular zone could not be determined due to the large macular hole. b Twelve months after vitrectomy, examination demonstrated increased retinal sensitivity in the area of the previous macular hole and its adjacent region. The postoperative preferred fixation position was located at the base of the previous macular hole and was different from the preoperative position. 


\section{References}

1 Thach AB, Lopez PF, Snady-McCoy LC, Golub BM, Frambach DA: Accidental Nd:YAG laser injuries to the macula. Am J Ophthalmol 1995;119:767-773.

-2 Potthöfer S, Foerster MH: Vitrectomy and autologous thrombocyte adhesion of an accidental macular hole caused by Nd:YAG laser. Br J Ophthalmol 1997;81:803-804

3 Chuang LH, Lai CC, Yang KJ, Chen TL, Ku WC: A traumatic macular hole secondary to a high-energy Nd:YAG laser. Ophthalmic Surg Lasers 2001;32:73-76.

-4 Hagemann LF, Costa RA, Ferreira HM, Farah ME: Optical coherence tomography of a traumatic Neodymium:YAG laser-induced macular hole. Ophthalmic Surg Lasers 2003;34:57-59.

-5 Sou R, Kusaka S, Ohji M, Gomi F, Ikuno Y, Tano Y: Optical coherence tomographic evaluation of a surgically treated traumatic macular hole secondary to Nd:YAG laser injury. Am J Ophthalmol 2003;135:537-559.

6 Ben Simon GJ, Nemet A, Moisseiev J: Vitrectomy with indocyanine green-guided inner limiting membrane peeling for a Q-switched Nd:YAG laser-induced macular hole. Retina 2003;23:714-716.

7 Gao L, Dong F, Chan WM: Traumatic macular hole secondary to Nd:YAG laser. Eye 2007;21:571-573.

-8 Ciulla TA, Topping TM: Surgical treatment of a macular hole secondary to accidental laser burn. Arch Ophthalmol 1997;115:929-930.

-9 Sakaguchi H, Ohji M. Kubota A, Otori Y, Hayashi A, Kusaka S, Saito Y, Tano Y: Amsler grid examination and optical coherence tomography of a macular hole caused by accidental Nd:YAG laser injury. Am J Ophthalmol 2000;130:355-356.

The author has no proprietary or commercial interest in any materials discussed in the article. 